\title{
HIGH-CYCLE FATIGUE OPTIMIZATION OF LAMINATED COMPOSITE STRUCTURES
}

\author{
Sebastian M. Hermansen $^{1 *}$, Erik Lund ${ }^{1}$ \\ ${ }^{1}$ Department of Materials and Production, Aalborg University, Fibigerstræde 16, 9220 Aalborg East, \\ Denmark \\ * smhe@mp.aau.dk
}

Structural design against fatigue failure is typically a comprehensive exercise. This is particularly the case for laminated composite structures due to the added complexity associated with their multi-directional behavior, resulting in material- and load-dependent failure modes. In fatigue analysis, these factors materialize as a non-linear relationship between reversals to failure and mean stress, load sequence effects as well as stiffness and strength degradation that also have to be taken into account.

To minimize the material use in composite structures, it is desirable to apply structural optimization. In this work, a methodology for gradient-based high-cycle fatigue optimization of general laminated composite structures is presented. An efficient approach for fatigue topology optimization was demonstrated in [1] by the use of an aggregation function to reduce the amount of fatigue damage measures from local finite element quantities to a single global measure. This is utilized with the adjoint method to efficiently compute gradients by solving only an extra set of linear equations where the factored stiffness matrix is reused. This same approach is adopted for solving the present problem. Parametrization of the structure is done by Discrete Material and Thickness Optimization (DMTO) approach [2], such that an optimized combination of material, fiber orientation, layup sequence, and layer thickness is sought.

The fatigue analysis approach used in this work is typically employed in the wind turbine industry for blade design, see e.g. [3]. Offset is taken in variable amplitude loading, which is quantified by Rainflow counting yielding a set of scaling factors for determining amplitude and mean stress. Proportional loading is assumed, meaning the computationally expensive Rainflow counting only has to be performed once during the optimization. A constant life diagram approach is used to calculate an equivalent stress from the amplitude and mean components, taking into account mean stress of various magnitudes by interpolating between their respective SN curves. Reversals to failure are then computed from the SN curves, which are constructed using a data-fitted power law. Damages are then summed using cumulative methods such as linear and non-linear Palmgren-Miner sum. A number of structural optimization examples including fatigue constraints will demonstrate the potential of this approach.

\section{References}

[1] Jacob Oest and Erik Lund (2017) Topology optimization with finite-life fatigue constraints. Structural and Multidisciplinary Optimization, 56, 1045-1059.

[2] Erik Lund (2018) Discrete Material and Thickness Optimization of laminated composite structures including failure criteria. Structural and Multidisciplinary Optimization, 57, 2357-2375.

[3] R.P.L. Nijssen (2006) Fatigue Life Prediction and Strength Degradation of Wind Turbine Rotor Blade Composites'. Ph.D. Thesis, Faculty of Aerospace Engineering, T.U. Delft 